\title{
Phase Separation and Flux Quantization in the Doped Quantum Dimer Model on Square and Triangular Lattices
}

\author{
Arnaud Ralko, ${ }^{1}$ Frédéric Mila, ${ }^{2}$ and Didier Poilblanc ${ }^{1}$ \\ ${ }^{1}$ Laboratoire de Physique Théorique, CNRS \& Université Paul Sabatier, F-31062 Toulouse, France \\ ${ }^{2}$ Institute of Theoretical Physics, Ecole Polytechnique Fédérale de Lausanne (EPFL), CH-1015 Lausanne, Switzerland \\ (Received 5 April 2007; revised manuscript received 3 July 2007; published 17 September 2007)
}

\begin{abstract}
The doped two-dimensional quantum dimer model is investigated by numerical techniques on the square and triangular lattices, with significantly different results. On the square lattice, at small enough doping, there is always a phase separation between an insulating valence-bond solid and a uniform superfluid phase, whereas on the triangular lattice, doping leads directly to a uniform superfluid in a large portion of the resonating-valence-bond (RVB) phase. Under an applied Aharonov-Bohm flux, the superfluid exhibits quantization in terms of half-flux quanta, consistent with $Q=2 e$ elementary charge quanta in transport properties.
\end{abstract}

DOI: 10.1103/PhysRevLett.99.127202

PACS numbers: 75.10.Jm, 05.30.- d, 05.50.+q

Understanding electron pairing in high temperature superconductors is a major challenge in strongly correlated systems. In his milestone paper, Anderson proposed a simple connection between high temperature superconductors and Mott insulators [1]. Electron pairs "hidden" in the strongly correlated insulating parent state as valence bond (VB) singlets lead, once fried to move at finite doping, to a superconducting behavior. A very good candidate of the insulating parent state is the resonating VB state (RVB), a state with only exponentially decaying correlations and no lattice symmetry breaking. A simple realization of RVB has been proposed by Rokhsar and Kivelson (RK) in the framework of an effective quantum dimer model (QDM) with only local processes and orthogonal dimer coverings [2]. Even though the relevance of these models for the description of SU(2) Heisenberg models is still debated, this approach is expected to capture the physics of systems that naturally possess singlet ground states (GS). For instance, specific quantum dimer models have recently been derived from a spin-orbital model describing $\mathrm{LiNiO}_{2}$ [3], or from the trimerized kagome antiferromagnet [4]. In a recent work, a family of doped QDMs (at $T=0$ ) generalizing the so-called RK point of Ref. [2] has been constructed and investigated [5], taking advantage of a mapping to classical dimer models [6] that extends the mapping of the RK model onto a classical model at infinite temperature, with evidence of phase separation at low doping. However, the soluble models of Ref. [5] are " $a d$ hoc" constructions, and this calls for the investigation of similar issues in the context of more realistic models. In that respect, a natural minimal model to describe the motion of charge carriers in a sea of dimers is the twodimensional quantum hard-core dimer-gas Hamiltonian:

$$
H=v \sum_{c} N_{c}|c\rangle\left\langle c\left|-J \sum_{\left(c, c^{\prime}\right)}\right| c^{\prime}\right\rangle\left\langle c\left|-t \sum_{\left(c, c^{\prime \prime}\right)}\right| c^{\prime \prime}\right\rangle\langle c|
$$

where the sum on $(c)$ runs over all configurations of the
Hilbert space, $N_{c}$ is the number of flippable plaquettes, the sum on $\left(c^{\prime}, c\right)$ runs over all configurations $|c\rangle$ and $\left|c^{\prime}\right\rangle$ that differ by a single plaquette dimer flip, and the sum on ( $c^{\prime \prime}$, c) runs over all configurations $|c\rangle$ and $\left|c^{\prime \prime}\right\rangle$ that differ by a single hole hopping between nearest neighbors (triangular) or (diagonal) next-nearest neighbors (square). Throughout, the energy scale is set by $J=1$. A schematic phase diagram for the two lattices is depicted in Fig. 1 in the undoped case. Remarkably, these lattices lead to quite different insulating states. Indeed, an ordered plaquette phase appears on the square lattice immediately away from the special RK point, whereas a RVB liquid phase is present in the triangular lattice.

In this Letter, we investigate in details the properties of model (1) on the square and triangular lattices at finite doping. Building on the differences between the two lattices in the undoped case, we investigate to which extent the properties of the doped system are governed by the nature of the insulating parent state. This investigation is based on exact Diagonalizations and extensive Green's Function Monte Carlo (GFMC) simulations [7] essentially free of the usual finite-size limitations [8].

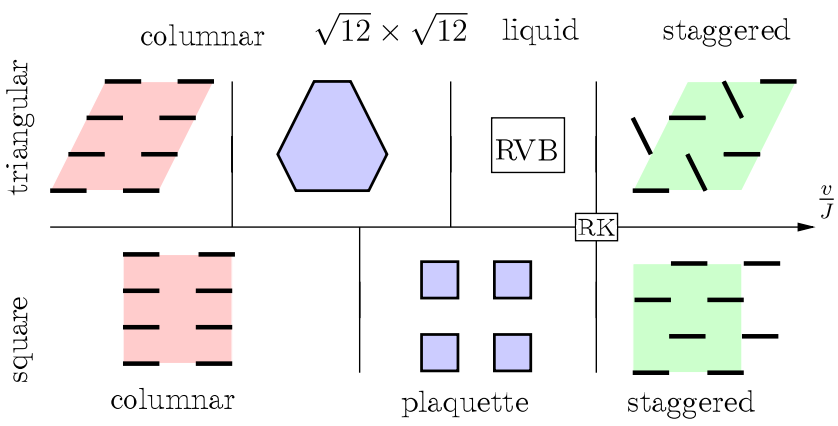

FIG. 1 (color online). Schematic phase diagrams for the triangular and the square lattice. 
Phase separation. - At small $t$, it is expected that holes experience an effective attractive potential. It is therefore natural to first address the issue of phase separation (PS), i.e., the possibility for the system to spontaneously undergo a macroscopic segregation into two phases with different hole concentrations. We analyze the problem as a function of the hopping parameter $t$ and hole concentration $x=$ $n_{h} / N$, where $n_{h}$ is the number of holes in the system and $N$ the number of sites. In order to perform a Maxwell construction, we define

$$
s(x)=\frac{e(x)-e(0)}{x}
$$

where $e(x)$ is the energy per site at doping $x$. This quantity corresponds to the slope of the line passing through $e(0)$ and $e(x)$. If the system exhibits PS, the energy will present a change of curvature implying $s(x)$ to have a minimum at a critical doping $x_{c}$. The fact that the local curvature of $e(x)$ at $x=0$ is negative then implies that the two separated phases will have $x=x_{c}$ and $x=0$ (the undoped insulator). In Fig. 2, typical results are shown for both square and triangular lattices and for different sizes.

Interestingly, PS appears in both cases, but with noticeable differences. While for the square lattice (lower panel) the critical hole concentration $x_{c}$ is roughly size independent, there is a strong size dependence for the triangular lattice (upper panel). This size effect can be traced back to the nature of the parent undoped state. On the square lattice, the crystalline phase (for $v<1$ ) at zero doping is very robust, and for increasing size, its local order changes only weakly. On the triangular lattice, it has been shown that size effects play an important role [8], especially in the RVB liquid phase for $0.8 \leq v \leq 1$. Periodic boundary conditions (BC) tend to stabilize the so-called $\sqrt{12} \times$ $\sqrt{12}$ phase on small clusters, and clusters with more than 192 sites are necessary to significantly reduce finite-size
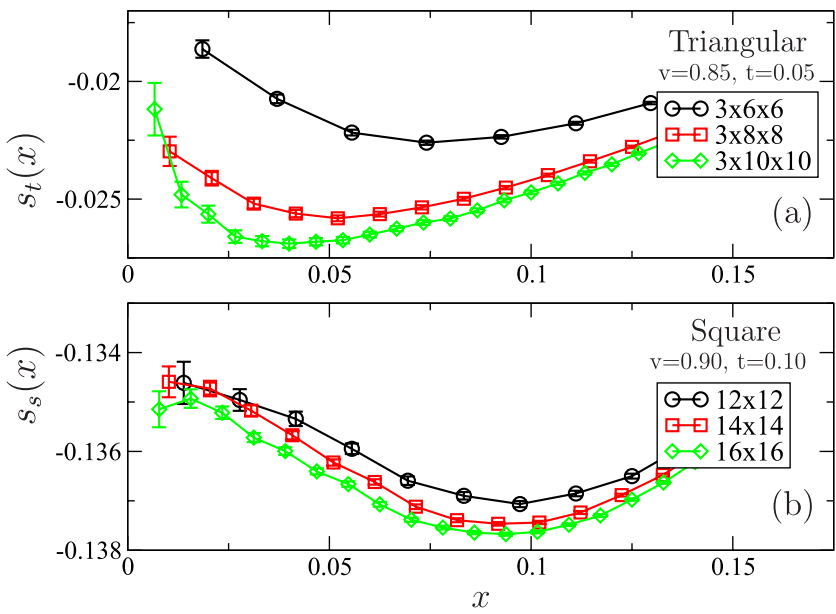

FIG. 2 (color online). Slope of energy density [Eq. (2)] vs doping for different sizes. (a) Triangular lattice. (b) Square lattice. effects, in particular, as in Fig. 2, close to the transition point with the crystalline phase. Hence, the PS observed around $x=0.075$ for the $3 \times 6 \times 6$ cluster is not representative of the thermodynamic limit.

To obtain the phase diagram in the $(v, x)$ plane, we have performed a systematic size-scaling analysis at fixed $t$ and for various $v$ 's depicted in Fig. 3.

In agreement with the previous discussion, a significant size dependence is only present for the triangular lattice, in which case PS disappears for large clusters in the RVB phase in the vicinity of the RK point [9]. In Fig. 4, we report the thermodynamic limit of $x_{c}$ for the two lattices as a function of $v$, and for different values of $t$. For the square lattice, calculations have been done from the RK point down to the expected phase transition between the plaquette phase and the columnar phase, namely $v \simeq 0.6$ [10]. For the triangular lattice, the range between the RK point down to the RVB- $\sqrt{12} \times \sqrt{12}$ transition point at $v \simeq 0.8$ has been covered [8].

These results clearly demonstrate the difference between the square and triangular lattices. In the first case, as soon as $v \neq 1$, PS occurs for $x<x_{c}$. Moreover, upon decreasing $v$, crystalline order strengthens, and, for fixed $t$, it is necessary to consider a higher concentration of holes to reach a stable conducting phase. Similarly, the bigger $t$, the lower $x_{c}$. On the triangular lattice, a finite size-scaling analysis shows that no phase separation appears down to a critical value $v \sim 0.9$, well above the critical value $v \sim 0.8$ below which plaquette order sets in. Although numerical limitations prevent computations for smaller $v$ and $t$, our results up to the $3 \times 12 \times 12$-site cluster provide clear evidence for a region of PS inside the RVB region, between $v \sim 0.8$ and $v \sim 0.9$ [11].

Dimer ordering on the square lattice. - Next, we investigate how dimer order, known to exist at $x=0$, evolves
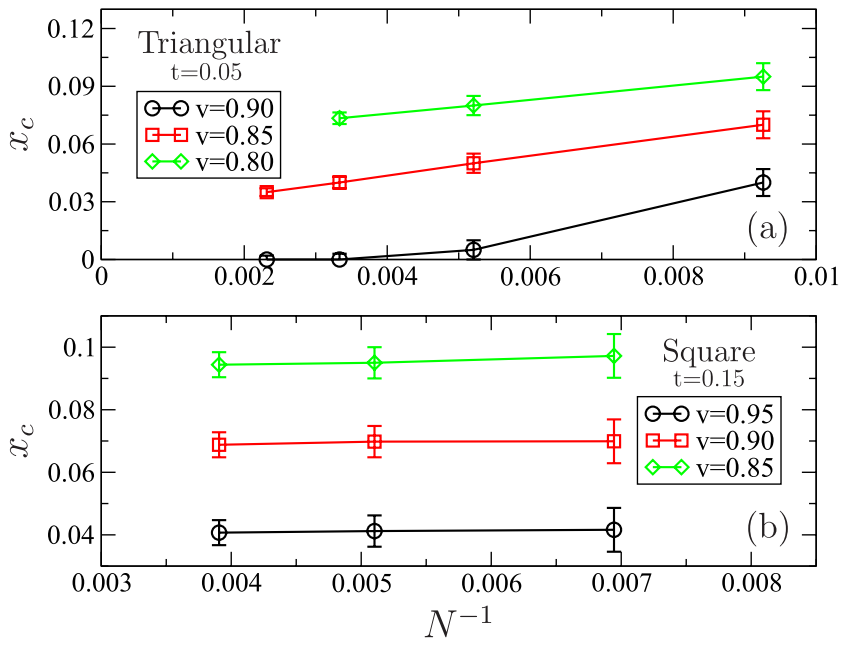

FIG. 3 (color online). Scaling of the critical doping $x_{c}$ defined by Maxwell construction with the inverse total number of sites. (a) Triangular lattice. (b) Square lattice. 


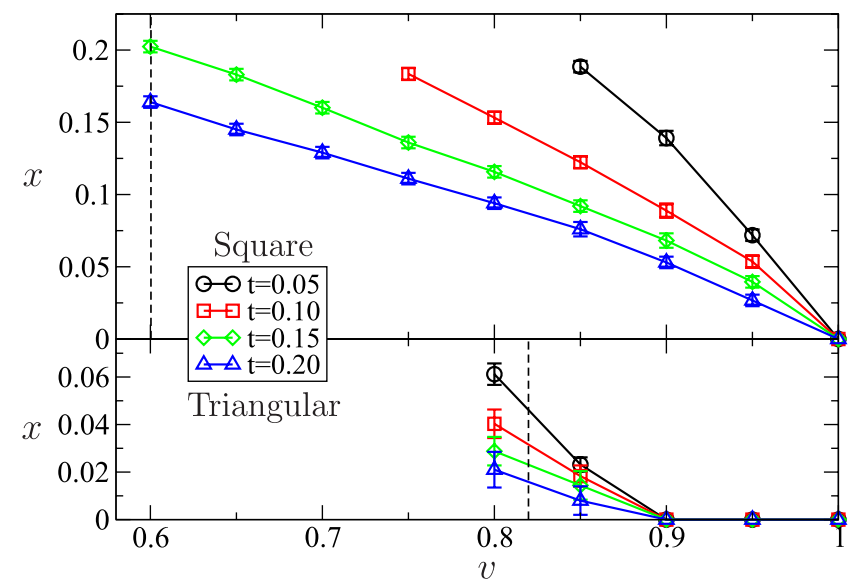

FIG. 4 (color online). Phase separation boundaries for the square and triangular lattices in the thermodynamic limit. The dashed lines correspond to the approximate location of the phase transition between plaquette and columnar phases [10] for the square lattice and between plaquette and RVB phases $[8,21]$ for the triangular lattice.

under finite doping. Two scenarii are a priori possible: (i) the dimer order vanishes in the stable conducting phase immediately at $x_{c}$; (ii) dimer order survives above $x_{c}$ in a narrow region of the conducting phase. To solve this problem, we have calculated the squared order parameter $D^{2}(\vec{k})$ in the GS $\left|\Psi_{0}\right\rangle$ defined by

$$
D^{2}(\vec{k})=\frac{1}{N} \frac{\left\langle\Psi_{0}|d(-\vec{k}) d(\vec{k})| \Psi_{0}\right\rangle}{\left\langle\Psi_{0} \mid \Psi_{0}\right\rangle}
$$

along the path of Fig. 5(a), the first Brillouin zone of the square lattice, where $d(\vec{k})$ is the Fourier transform of the dimer operator defined on the horizontal bonds. Note that this calculation has not been tried for the triangular lattice since no Bragg peak is present in the RVB phase, and the algorithm is losing efficiency for $v \lesssim 0.8$ [9]. In the pure

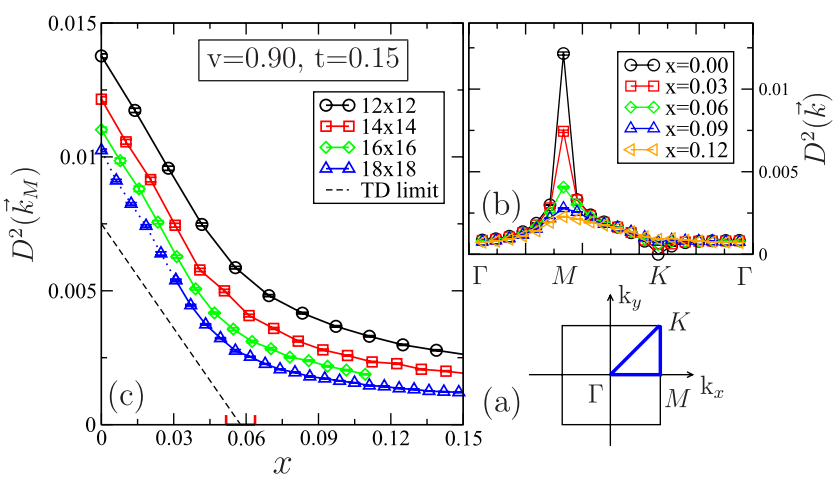

FIG. 5 (color online). (a) First Brillouin zone. (b) Momentum dependence of the squared order parameter for the 196-site cluster $(14 \times 14)$. (c) Squared order parameter at the $M$ point as a function of the hole concentration $x$ and for different cluster sizes. The thermodynamic limit is depicted as a dashed line, with the corresponding error bar (see main text). plaquette phase on the square lattice, a Bragg peak develops at point $\vec{k}_{M}=(\pi, 0)$, the middle of the side of the BZ. We show in Fig. 5(b) a typical result for the squared order parameter on the 196-site cluster for different values of $x$. Clearly, the Bragg peak disappears upon doping.

A finite size scaling of the order parameter can be performed thanks to the linear behavior at low concentration. Within our data, $D^{2}\left(\vec{k}=\vec{k}_{M}\right) \equiv D_{M}^{2}(L, x)$ behaves like $a_{L} x+b_{L}$ in the linear region. In this case, one can determine rather precisely $x_{+\infty}$ such that $D_{M}^{2}\left(+\infty, x_{+\infty}\right)=$ 0 , i.e., $x_{+\infty}$ is the concentration in the thermodynamic limit where the Bragg peak vanishes. By definition, $x_{+\infty}=$ $-b_{+\infty} / a_{+\infty} \simeq 0.05(8)$. This value, and the linear behavior in the thermodynamic limit, are displayed in Fig. 5(c). If we compare $x_{+\infty}$ to the corresponding $x_{c}$ from Fig. 4, we can conclude that the Bragg peak indeed vanishes at $x_{c} \simeq$ $0.067(5)$ within error bars. Note that numerical errors increase for larger clusters, and we are not able to use the same analysis for clusters larger than 256 sites (the results for the $18 \times 18$ cluster have not been used). Although the determination of $x_{+\infty}$ is delicate, the linear behavior of $D^{2}$ vs $x$ is consistent with the physical behavior expected for the binary system of Fig. 4. No dimer order is present above $x_{c}$, showing that the system is simply "conducting" in this case, with $D^{2}\left(\vec{k}_{M}\right)$ decreasing as $N^{-1}$ (critical behavior).

Flux quantization. - Finally, let us turn to a better characterization of the "conducting phase." Since holes are bosonic, one expects the conducting phase to be a superfluid (through Bose condensation) [12]. However, extra complexity results from the fact that these bosons move in a dimer environment. To investigate this issue, we pierce the torus by an Aharonov-Bohm flux of strength $\phi=\xi \phi_{0}$ with $0 \leq \xi \leq 1$ and where $\phi_{0}=h c / e$ is the magnetic flux quantum. To reduce finite-size effects, we also consider arbitrary BC in the second direction $(y)$. All this is implemented by the Peierls substitution, changing the hole hopping term into $t^{\prime}=\exp \left( \pm i 2 \pi \xi a / L_{x} \pm i 2 \pi \eta a / L_{y}\right) t$, where the \pm depends on the directions $\pm x \pm y$, while $a$ is the lattice parameter and $L_{x}$ and $L_{y}$ the linear sizes of the system. Obviously, the whole spectrum should be periodic in $\xi$ with period 1. We show in Fig. 6 the spectrum of the $4 \times 4$ cluster on the square lattice, with 4 holes.

It turns out that (i) the GS energy exhibits well-defined minima and (ii) is rigorously periodic with period $\xi=1 / 2$, which means that there is flux quantization in units of half the flux quantum (square symbols). Property (i) is typical of a superfluid [13]: It is the precursor of a finite barrier in the thermodynamic limit [14]. Property (ii) was suggested quite some time ago in the context of a more general QDM by Kivelson [12], who also predicted that, in the cylinder geometry, one should be able to tunnel between the two branches of Fig. 6, thus lifting the degeneracy at the level crossing. This degeneracy is not lifted in our case, neither in the torus geometry, nor in the cylinder geometry, due to the translational symmetry, which puts the two states that 


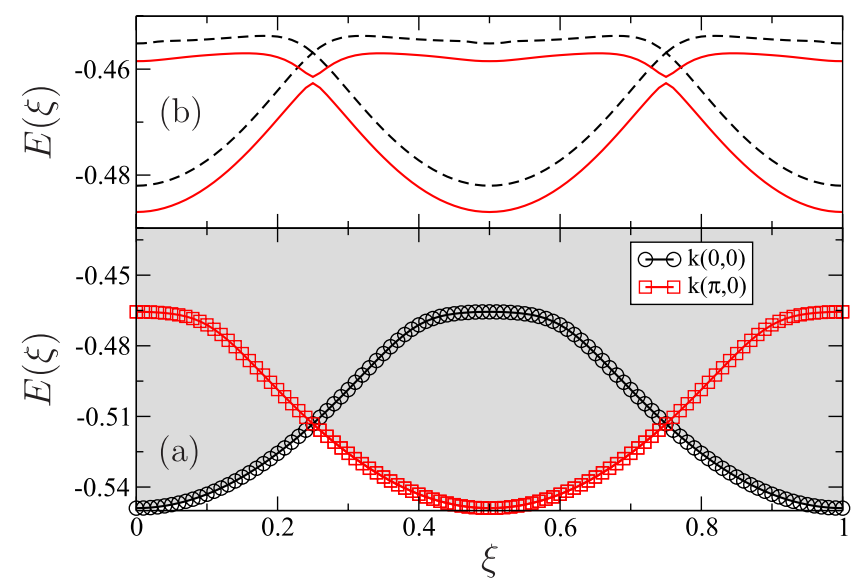

FIG. 6 (color online). Energy spectrum vs (reduced) Aharonov-Bohm flux $\xi$, for a $4 \times 4$ cluster with 4 holes at $v=$ 0.70 and $t=1.00$. (a) Torus geometry. Arbitrary BC are used in the transverse direction to obtain a continuous set of momenta leading to a continuum (shaded area). (b) The two low-energy branches for the case of a cylinder (dashed line) and including a small bond disorder (solid line).

are degenerate at $\xi=1 / 4$ into different symmetry sectors. However, getting rid of the translational symmetry by changing the amplitude of a local dimer flip indeed removes the degeneracy (upper panel of Fig. 6), leading to a detectable flux quantization in units of half the flux quantum in an experiment in which the flux is sweeped. Thus, in our model, the ground-state energy has periodicity $h c / 2 e$, consistent with mobile elementary particles of charge $Q=$ $2 e$ in the system. Unlike what was recently found in a bosonic model with correlated hopping [15], these particles are not boson pairs: From the bosonic point of view, it is the statistical flux of the dimer background that leads to the half-flux quantization. If dimers are interpreted as SU(2) electron singlets, these singlets are the physical pairs that lead to half-flux quantization. This scenario is fundamentally different from the usual mechanism related to real space pairing of the charge carriers found, e.g., in the extended Hubbard chain [16], in the 2-leg ladders [17], or, more generally, in Luther-Emery liquids [18], as can be inferred from the exact degeneracy between $\xi=0$ and $\xi=0.5$ for finite systems in the present case, to be contrasted with the significant finite-size effects of the other cases.

Summary and conclusions. - The numerical investigation with Green's function Quantum Monte Carlo and exact diagonalizations of the doped two-dimensional quantum hard-core dimer model on the square and triangular lattices has led to a number of interesting conclusions regarding hole motion in a dimer background. Phase separation is often present at low doping, as suggested by earlier investigations, but our results indicate that it is related to the presence of valence-bond order [19]: In the
RVB phase of the triangular lattice, PS only occurs close to the plaquette phase, where short-range dimer correlations are already strong enough. Close to the RK point, doping the RVB phase leads directly to a superfluid phase as shown from its response to an Aharonov-Bohm flux. Moreover, we observed that the flux quantization is in units of half a flux quantum, consistent with the idea that the dimer background leads to effective particles of charge $2 e$. All these results are in qualitative agreement with the gauge theories of high $T_{c}$ superconductivity in strongly correlated systems [20].

We acknowledge useful discussions with Federico Becca. This work was supported by the Swiss National Fund, by MaNEP, and by the Agence Nationale de la Recherche (France).

[1] P. W. Anderson, Science 235, 1196 (1987).

[2] D. S. Rokhsar and S. A. Kivelson, Phys. Rev. Lett. 61, 2376 (1988).

[3] F. Vernay, A. Ralko, F. Becca, and F. Mila, Phys. Rev. B 74, 054402 (2006).

[4] M.E. Zhitomirsky, Phys. Rev. B 71, 214413 (2005).

[5] D. Poilblanc, F. Alet, F. Becca, A. Ralko, F. Trousselet, and F. Mila, Phys. Rev. B 74, 014437 (2006).

[6] C. Castelnovo, C. Chamon, C. Mudry, and P. Pujol, Ann. Phys. (Leipzig) 322, 903 (2007).

[7] N. Trivedi and D. M. Ceperley, Phys. Rev. B 41, 4552 (1990); M. Calandra and S. Sorella, Phys. Rev. B 57, 11446 (1998).

[8] A. Ralko, M. Ferrero, F. Becca, D. Ivanov, and F. Mila, Phys. Rev. B 74, 134301 (2006), and references therein.

[9] For small values of $t$, the GFMC algorithm is no longer ergodic due to hole localization.

[10] O.F. Syljuasen, Phys. Rev. B 73, 245105 (2006).

[11] Long-range Coulomb repulsion is expected to have a major role in the PS region and might stabilize stripes.

[12] S. Kivelson, Phys. Rev. B 39, 259 (1989).

[13] N. Byers and C. N. Yang, Phys. Rev. Lett. 7, 46 (1961).

[14] By contrast, for noninteracting fermions, signs of a flat energy curve already appear on such small clusters provided one also uses arbitrary BC. Similar arguments were used in D. Poilblanc, Phys. Rev. B 44, 9562 (1991) for the 2D $t$ - $J$ model.

[15] R. Bendjama, B. Kumar, and F. Mila, Phys. Rev. Lett. 95, 110406 (2005).

[16] K. Penc and F. Mila, Phys. Rev. B 49, 9670 (1994).

[17] C. A. Hayward, D. Poilblanc, R. M. Noack, D. J. Scalapino, and W. Hanke, Phys. Rev. Lett. 75, 926 (1995).

[18] A. Seidel and D. H. Lee, Phys. Rev. B 71, 045113 (2005).

[19] Our results give an explicit characterization of the confinement-deconfinement discussed in O.F. Syljuasen, Phys. Rev. B 71, 020401(R) (2005).

[20] T. Senthil and P. A. Lee, Phys. Rev. B 71, 174515 (2005), and references therein.

[21] R. Moessner and S. L. Sondhi, Phys. Rev. B 63, 224401 (2001). 